\title{
Hamartoma lipofibromatoso de nervio mediano. \\ Caso clínico y revisión bibliográfica
}

\author{
M. Alvarez ${ }^{(1)}$, N. Cortabarria $^{(2)}, J$. Manzani ${ }^{(3)}$, R. Graciano $^{(2)}$ \\ CÁtedra de Cirugía Plástica, Reparadora y Estética. Hospital de Clínicas. Montevideo - Uruguay \\ (1) MÉdico RESIDENTE \\ (2) MÉdico AsISTENTE \\ (3) PROFESOR ADJUNTO
}

Correspondencia:

Dr. Manuel Alvarez Antiga

Avenida Italia $\mathrm{s} / \mathrm{n}$

Distrito postal 11600. Montevideo, Uruguay

Teléfono 598(2)4874521

e-mail: Dralvarezantiga@gmail.com

\begin{abstract}
El hamartoma de nervio mediano es una neoplasia beniga poco frecuente, que afecta predominantemente al nervio mediano de pacientes jóvenes, y se manifiesta generalmente por sintomatología compresiva distal a nivel del mismo, acompañado o no de macrodactilia.

Actualmente se conocen elementos del estudio por imagen patognomónicos, pero continúa siendo un hallazgo intraoperatorio frecuentemente. El tratamiento quirúrgico principal está dirigido únicamente a la descompresión nerviosa, dado los riesgos de lesión nerviosa por resección total o parcial mediante disección interfascicular.

Realizamos la descripción de un caso clínico con compromiso del nervio mediano, sin macrodactilia asociada, que constituyó un hallazgo intraoperatorio y en el cual se realizó una resección parcial de la tumoración, obteniendo una mejoría funcional significativa con una mínima secuela, en relación a su estado previo y actividad. Actualmente este caso tiene un año de evolución postoperatoria sin recidiva. Se realizó un análisis bibliográfico del tema.
\end{abstract}

Palabras clave: hamartoma lipofibromatoso, nervio mediano.
The hamartome or median nerve are benign rare neoplasia, predominantly affecting the median, nerve in young patients, and is usually manifested by distal compressive symptoms in it, accompanied or not macrodactilia. Elements of the study by image are pathognomonics, but continues to be an intraoperative finding frequently are currently known. Surgical treatment main this aimed solely at nerve decompression, given the risks of nerve injury by total or partial resection using interfascicular dissection. We conducted a clinical case with engagement without associated macrodactilia median, nerve, which constituted an intraoperative finding and which made a partial resection of the tumor, obtaining significant functional improvement with minimum sequel, regarding its previous state and activity description. Actually this case have one year postoperatory evolution without recidive A bibliographic item analysis was conducted

Key word: fibrolipomatosis hamartome, median nerve. 


\section{CASO CLÍNICO}

$\mathrm{P}$ aciente de 55 años, diestra, comerciante, obesa, con antecedentes personales de ex fumadora; hipotiroidismo desde 1998 secundario a tratamiento con radioyodo por hipertiroidismo, actualmente eutiroidea; ablación de vía accesoria cardiaca en 1999; liberación de neuropatía compresiva distal en canal carpiano derecho en 1999, con remisión completa de sintomatología. Consulta en junio del año 2009 por aumento difuso del volumen de la eminencia tenar derecha de un año de evolución, progresivo, que dificulta la prensión e imposibilita sus actividades diarias. Niega traumatismos previos ni infecciones. Se acompaña de dolor y parestesias irradiadas a los tres primeros dedos radiales, hipoestesia predominantemente de $1^{\text {er }}$ y $2^{\circ}$ dedos, con episodios de caída de objetos pequeños.

$\mathrm{Al}$ examen se constata a nivel de cara palmar de mano y muñeca derecha: tumoración difusa de eminencia tenar, blanda, ovoidea, de $4 \mathrm{~cm}$ de diámetro, sin límites netos, incluida en la musculatura tenar, no dolorosa acompañada de otra tumoración longitudinal de $3 \mathrm{~cm}$ a nivel de cara anterior de muñeca, central, de bordes poco netos, de superficie lisa, con signo de Tinel presente y maniobra de Phalen positiva.

Limitación de antepulsión del pulgar e imposibilidad de oposición completa del pulgar. Fuerza conservada.

Cicatriz de abordaje de liberación de canal carpiano previa, madura e indolora.

No otras tumoraciones, no máculas faciales ni axilares, no nódulos subcutáneos.

Planteamiento clínico: Tumoración de partes blandas de eminencia tenar y cara anterior de muñeca con recidiva de neuropatía compresiva distal de nervio mediano, presunción de tumoración de nervio mediano.

Se solicitan los siguientes exámenes complementarios: radiografía simple de mano derecha, proyecciones frente y perfil, que informa: $\mathrm{marca}$ da osteopenia por desmineralización ósea difusa y edema de partes blandas de eminencia tenar. Ecografía de partes blandas mano derecha con equipo de baja resolución para partes blandas que informa: área de eco negativa laminar de $30 \mathrm{~mm}$ x $6 \mathrm{~mm}$ que podría corresponder a líquido, no imágenes sólidas ni quísticas bien definidas.

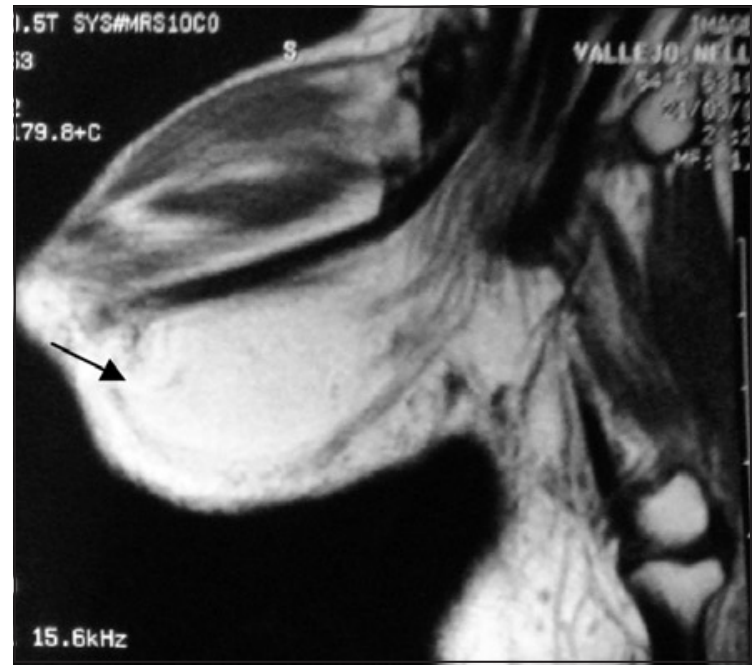

Figura 1. RM. Lipoma de eminencia tenar que infiltra planos musculares.

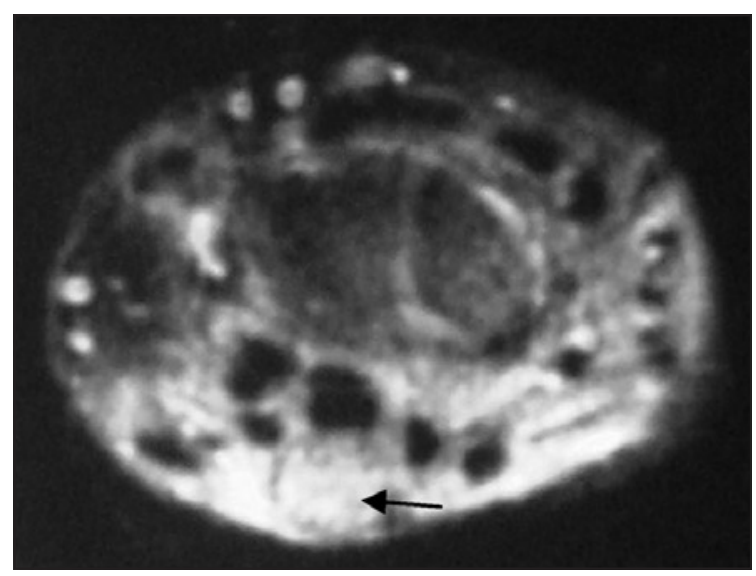

Figura 2. RM. Sin evidencia de sufrimiento de nervio mediano.

Dada la sintomatología nerviosa se valora con un estudio electrofisiológico de miembros superiores que muestra denervación parcial de mediana entidad en territorio de inervación distal de nervio mediano derecho compatible con una neuropatía del nervio mediano derecho sensitivo-motora por probable compresión en canal carpiano.

Orientados con el estudio ecográfico, no concluyente, y para el mejor estudio de las tumoraciones de partes blandas es solicitada una resonancia magnética (RM), que informa: segmentos óseos de morfología y señal normal; ausencia de lesiones tendido-ligamentarías; y la presencia de lipoma de eminencia tenar que infiltra planos musculares (Figura 1); sin evidencia de sufrimiento de nervio mediano (Figura 2). 


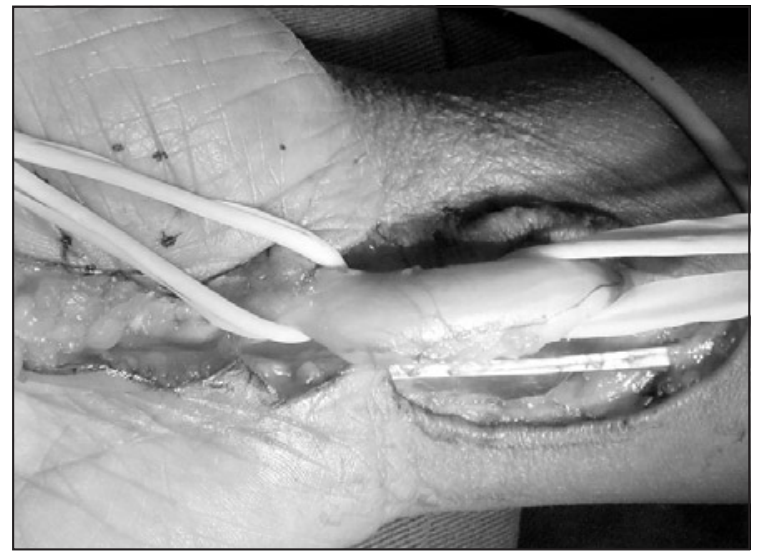

Figura 3. Se amplía la incisión al antebrazo.

Se decide realizar tratamiento quirúrgico mediante abordaje de eminencia tenar, para el tratamiento del lipoma y del canal carpiano, para exploración y eventual resección tumoral que pudiera ser la causa de la compresión.

Se aborda primeramente la celda tenar constatando una tumoración amarillo grisácea de aspecto fibroadiposo. A continuación se realiza apertura del canal carpiano identificando aumento de tamaño del nervio mediano de manera difusa. Se amplía incisión al tercio distal del antebrazo, sin identificar sectores del nervio sanos proximalmente (Figura 3). A nivel distal se identifica engrosamiento de las ramas colaterales digitales del nervio y de la rama tenar.

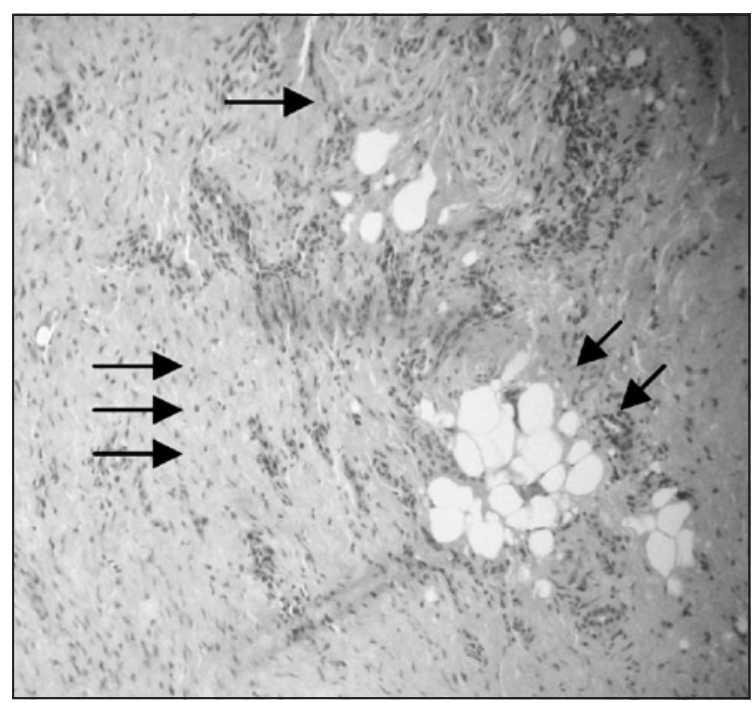

Figura 4. Anatomía patológica: células tipo fibroblastos y tejido adiposo.

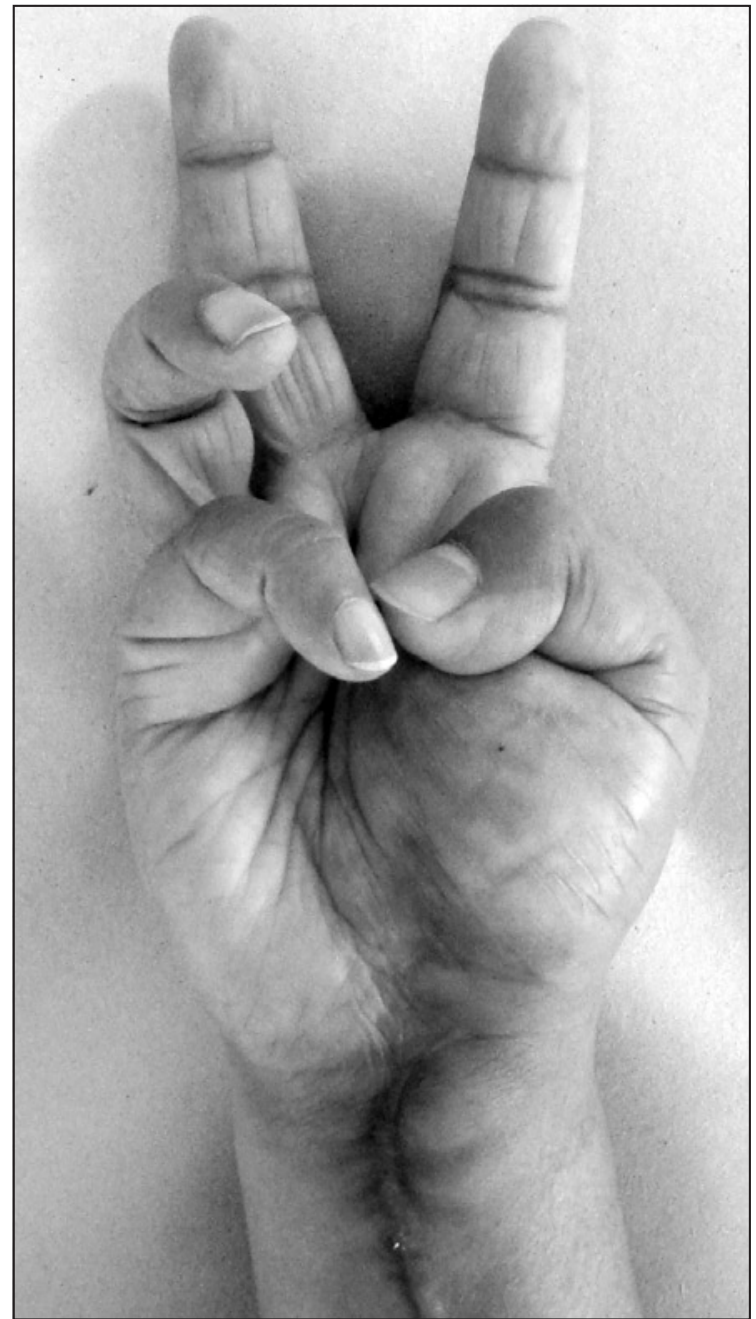

Figura 5. Buena función global de la mano.

Se procede a resección de tumoración tenar y toma de biopsia de sector dorsal del nervio con técnica microquirúrgica evitando realizar disección interfascicular extensa.

El informe de anatomía patológica informa como: proliferación mesenquimática benigna con células tipo fibroblastos y tejido adiposo alrededor de fibras nerviosas con caracteres de neurofibrolipoma (Figura 4).

En la evolución, al año de la intervención, se presenta con remisión completa de la sintomatología nerviosa irritativa, recuperación de la oposición del pulgar e hipoestesia en hemipulpejo cubital del pulgar con una buena función global de la mano (Figura 5), con lo cual la paciente se reintegra satisfactoriamente a sus actividades cotidianas. 


\section{DISCUSIÓN}

El hamartoma es una proliferación de tejido desorganizado compuesto por células inherentes al tejido implicado ${ }^{1}$. El hamartoma lipofibromatoso es una neoplasia intraneural benigna extremadamente rara, caracterizada por un sobrecrecimiento del tejido fibroadiposo epineural ${ }^{2}$.

Se han referido varios sinónimos para esta patología: lipoma intraneural, lipofibroma nervioso, lipofibromatosis neural, hamartoma lipofibromatoso, hamartoma nervioso, proliferación fibroadiposa, siendo la más aceptada hamartoma lipofibromatoso ${ }^{3}$. Según la clasificación de la OMS corresponde a lipomatosis del nervio ${ }^{4}$.

Se atribuye su primer descripción a Mason en $1953^{5}$. Hasta el año 2005 se publicaron 175 casos, de los cuales 125 corresponden a compromiso del nervio mediano ${ }^{6}$.

$\mathrm{Su}$ etiología es desconocida, atribuyéndose una causa congénita ${ }^{2}$, irritación crónica del nervio o antecedentes de traumatismo ${ }^{3}$.

Se presenta más frecuentemente en la niñez o antes de los 30 años, pero se han descrito casos después de los 50 años ${ }^{7}$. En un tercio de los casos se acompaña de macrodactilia ${ }^{2}$, principalmente en los dedos que corresponden al territorio sensitivo del nervio; cuando se presenta con una macrodactilia verdadera se denomina macrodistrofia lipomatosa.

Es más frecuente en el sexo femenino cuando se presenta con macrodactilia, sin macrodactilia la proporción es similar en ambos sexos ${ }^{2,8}$.

El nervio más frecuentemente afectado es el nervio mediano, también se han descrito compromiso del nervio cubital ${ }^{2,9}$, la rama superficial del nervio radial ${ }^{10}$, el nervio peroneo superficial $^{11}$, el nervio ciático ${ }^{12}$, el plexo braquial $^{13} \mathrm{y}$ raramente un par craneal ${ }^{14}$.

Macroscópicamente se reconoce una masa amarillenta, blanda, lisa, que corresponde a un nervio aumentado de diámetro y longitud difusamente, sin adherencia a los tejidos que lo rodean.

Histológicamente hay una proliferación de tejido fibroadiposo maduro que separa y comprime los fascículos nerviosos e infiltra el epineuro. Puede haber atrofia axonal y fibrosis perineural ${ }^{3}$. También se ha descrito hueso heterotópico ${ }^{15}$.

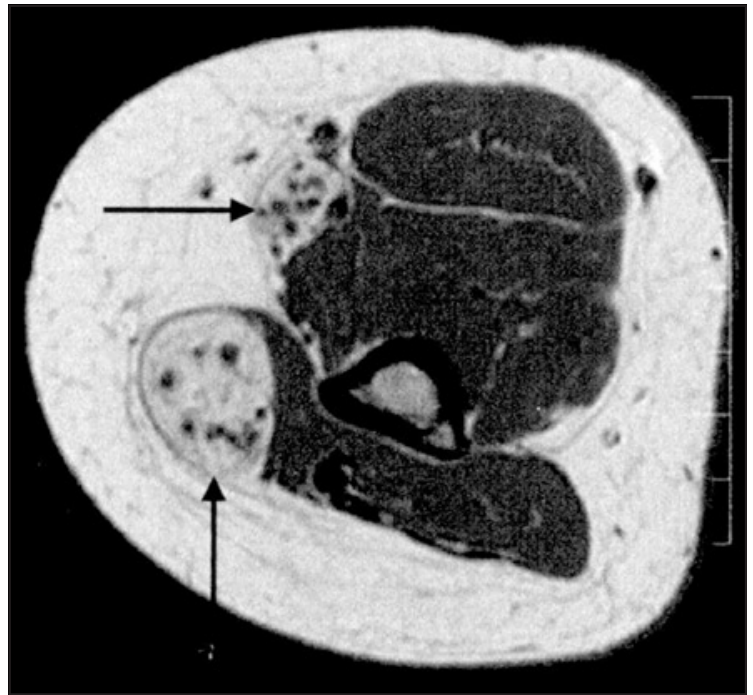

Figura 6. RM. Imagen en espagueti.

El diagnóstico diferencial corresponde a lipoma intraneural, neuroma, schwannoma, neurofibroma y ganglión de la vaina del nervio.

Clínicamente se presenta en su mayor frecuencia como una tumoración en la cara palmar de la muñeca o tercio distal de antebrazo, de lento crecimiento, acompañado o no de macrodactilia, en un niño o adulto joven, con sintomatología nerviosa compresiva distal concomitantemente ${ }^{16}$.

El diagnóstico surge de los pilares clínicos y de imagen, con confirmación anatomopatológica por un anatomopatólogo con experiencia ${ }^{2}$.

La RM es el «gold standard» para el estudio de esta patología. Confirma el diagnóstico mostrando fascículos nerviosos serpentiformes rodeados por tejido fibroadiposo dentro de un vaina neural aumentada de tamaño, dispuestos asimétricamente; y permite completar la valoración preoperatoria del nervio comprometido. En los cortes coronales la imagen patognomónica corresponde a la imagen en espagueti ${ }^{17}$ (Figura 6).

Se pueden realizar como estudios complementarios: estudio electrofisiológico de miembros superiores; y radiografía simple de manos, principalmente en pacientes con macrodactilia.

El tratamiento es controvertido, contamos con tratamiento conservador, neurolísis externa o interna, para resección total o parcial de la tumoración, y escisión radical con o sin injertos fasciculares. 
El más comúnmente elegido es el tratamiento conservador, que consiste en la liberación del nervio de todos los sitios de compresión. Las razones corresponden al riesgo de déficit neurológico, posibilidad de dolor neurogénico en el postoperatorio y a que el compromiso nervioso puede llegar hasta el plexo $^{2,11}$.

\section{CONCLUSIONES}

El hamartoma lipofibromatoso es un raro tumor intraneural benigno que a pesar de los adelantos de los estudios por imagen, como en este caso, puede llegar ser un hallazgo intraoperato- rio. Por lo tanto se debe mantener su sospecha a la hora de asistir a un paciente con una tumoración de miembro superior, principalmente en sus segmentos dístales, y una neuropatía compresiva asociada. Así como contar con un equipo entrenado en microcirugía nerviosa.

Si bien se propone un tratamiento conservador para casos clínicos similares, en nuestra paciente, la resección de la masa tumoral principal y liberación del nervio mediano determinó una franca mejoría en la sintomatología irritativa y así en la función global de la mano. Esto con una secuela mínima aceptable desde el punto de vista funcional, en relación a su actividad y a su estado previo.

\section{BIBLIOGRAFÍA}

1. Nardella D, Sohawon S, Carlier A. Lipofibromatous hamartoma of the median nerve. Three case reports. J Plast Reconstr Aesthet Surg, 2009; 62: e314-7.

2. Silverman TA, Enzinger FM. Fibrolipomatous hamartoma of nerve. A clinico-pathologic analysis of 26 cases. Am J Surg Pathol, 1985; 9: 7-14.

3. Razzaghi A, Anastakis DJ. Lipofibromatous hamartoma: review of early diagnosis and treatment. Can J Surg, 2005; 48: 394-9.

4. Fletcher CDM, Unni K, Mertens K. WHO classification of tumours. Pathology and genetics of tumours of soft tissue and bone. Lyon: IARC Press, 2002: $24 \mathrm{e} 5$.

5. Mason ML. Three cases of tumor of the hand. Proceedings of the 1952 annual meeting of the American Society of Surgery of the Hand. J Bone Joint Surg Am, 1953; 35: 273-4.
6. Bisceglia M, Vigilante E, BenDor D. Neural lipofibromatous hamartoma. A report of two cases and review of the literature. Adv Anat Pathol, 2007; 14: 46-52.

7. Kameh DS, Perez-Berenguer JL, Peral GS. Lipofibromatous hamartoma and related peripheral nerve lesions. South Med J, 2000; 93: 800-2.

8. Amadio PC., Reiman HM, Dobyns JH. Lipofibromatous hamartoma nerve. J Hand Surg Am, 1988; 13: 65-75.

9. Arlandis Villarroya S, Lajara Marco F, Fuentes Díaz A, et al. Fibrolipoma del nervio cubital. A propósito de un caso. Rev Iberamer Cir Mano, 2008; 36: 64-7.

10. Jacob RA, Buchino JJ. Lipofibroma of the superficial branch of the radial nerve. J Hand Surg Am, 1989; 14: 704-6.

11. Hirakawa E, Miki $\mathrm{H}$, Kobayashi S. Lipofibromatous hamartoma of nerve in the foot. Acta Pathol Jpn, 1993; 43: 265-7.

12. Fandridis EM, Kiriako AS, Spyridonos SG, et al. Lipomatosis of the sciatic nerve report of a case and review ofthe literature. Microsurgery, 2009; 29: 66-71.

13. Price AJ, Compson JP, Calonje E. Fibrolipomatous hamartoma of nerve arising in the brachial plexus. J Hand Surg Br, 1995; 20: 16-8.

14. Berti E, Roncaroli F. Fibrolipomatotosis hamartoma of a cranial nerve. Histopathology, 1994; 24: 391-2.

15. Lois DS, Dick HM. Ossifying lipofibroma of the median nerve. J Bone Joint Surg Am, 1973; 55: 1082-4.

16. Louis DS. Peripheral nerve tumors in the upper extremity. Hand Clin, 1987; 3: 311-8.

17. Marom EM, Helms CA. Fibrolipomatous hamartoma: pathognomonic on MR imaging. Skeletal Radiol, 1999; 28: 260-4. 